\title{
Asymptotic behaviors of the semigroup of the linearized Landau operator for the very soft and Coulomb potentials
}

\author{
Jiawei $\mathrm{SUN}^{1}$ and Yakui WU $\mathrm{W}^{2}$ \\ ${ }^{1}$ Institute of Applied Physics and Computational Mathematics \\ ${ }^{2}$ Capital Normal University
}

January 27, 2021

\begin{abstract}
We study the asymptotic behaviors of the semigroup generated by the linearized Landau operator in the case of the very soft potentials and Coulomb potential. Compared with the hard potentials, Maxwellian molecules and moderately soft potentials, there is no spectral gap for the linearized Landau operator with the very soft and Coulomb potentials. By introducing a new decomposition of the linear Landau collision operator $\$ \mathrm{~L} \$$ including an accretive operator and a relatively compact operator, we establish the complete spectrum structure for the linearized Landau operator with the very soft and Coulomb potentials and furthermore derive the time decay estimates of the corresponding semigroup in a weighted velocity space.
\end{abstract}

\section{Hosted file}

Manuscript.pdf available at https://authorea.com/users/392039/articles/506051-asymptoticbehaviors-of-the-semigroup-of-the-linearized-landau-operator-for-the-very-soft-andcoulomb-potentials 